\title{
Etude comparative des performances des plateformes de compostage de Siteu et de Ngui à Dschang (Cameroun)
}

\author{
Ngahane Emilienne Laure, PhD \\ Université de Dschang, Faculté d'Agronomie et des Sciences Agricoles, \\ Filière des Métiers du Bois, de l'Eau et de l'Environnement \\ (UDs/FASA/FMBEE) \\ Sagne Moubé Joël, PhD \\ Université de Dschang, Faculté des Lettres et des Sciences Humaines, \\ Département de Géographie (UDs/FLSH/DG) \\ Nana Paulin, PhD \\ Université de Dschang, Faculté d'Agronomie et des Sciences Agricoles, \\ Filière des Métiers du Bois, de l'Eau et de l'Environnement \\ (UDs/FASA/FMBEE)
}

Doi:10.19044/esj.2021.v17n17p248

Submitted: 12 April 2021

Accepted: 11 May 2021

Published: 31 May 2021
Copyright 2021 Author(s)

Under Creative Commons BY-NC-ND

4.0 OPEN ACCESS

Cite As:

Laure N.E., Joël S.M. \& Paulin N. (2021). Etude comparative des performances des plateformes de compostage de Siteu et de Ngui à Dschang (Cameroun). European Scientific Journal, ESJ, 17(17), 248. https://doi.org/10.19044/esj.2021.v17n17p248

\section{Résumé}

A Dschang, le compostage artisanal est le mode de traitement de la fraction fermentescible majoritaire dans les déchets locaux. Qualifié d'activité à haute intensité de main d'œuvre (HIMO), il permet de créer trois emplois directs par tonne/jour de déchets traités. Les deux plateformes fonctionnelles de nos jours à Dschang occupent une quarantaine de personnes et permettent de traiter en moyenne 3000 tonnes de déchets chaque année. Afin d'entrevoir leur autonomie, les équipes ont été évaluées par la méthode du bilan main d'œuvre. Cette méthode est pratique et permet d'exprimer la productivité des agents afin d'évaluer le coût de production et d'identifier les leviers d'optimisation. En ce sens, l'augmentation des quantités de déchets traités par agent afin de diminuer le coût de production et l'amélioration de la qualité de compost produit afin de faciliter son utilisation ont été identifiées comme les leviers internes. Cependant, s'il est aisément admis que la qualité obtenue est 
surtout conditionnée par la nature des déchets initiaux ; la quantité obtenue l'est tout aussi. En effet, l'étape de tri ainsi que l'étape d'affinage (criblage), nécessaires pour l'obtention d'un compost de qualité, représentent aussi les étapes les plus chronophages et qui déterminent objectivement leur productivité. A Dschang, ces deux étapes représentent en moyenne 84\% (66\% pour le tri et $18 \%$ pour le criblage) du temps de travail. Les performances de compostage mesurées diffèrent de $22 \%$ en faveur de Ngui où la productivité se situe à 10,41 ttraités/homme.mois tandis qu'elle se situe à 8,53 $\mathrm{t}_{\text {traitées }}$ homme.mois à Siteu. Les facteurs majeurs justifiant ces variations sont la qualité des déchets entrants (à 46,2\%) et l'aménagement des sites (à 34,3\%). Aussi, quelques retours négatifs, liés à la présence d'indésirables, ont été enregistrés auprès des utilisateurs du compost produit à Siteu. Donc, du fait de la meilleure qualité des déchets entrants et de la concentration des activités sur un espace limité à Ngui par rapport à Siteu, la consolidation des emplois créés et la pérennisation de l'activité de compostage seraient assurées sur le site de Ngui car il a été établi que ces dernières passeraient, entre autres, par l'atteinte d'une productivité minimale de $10 \mathrm{t}_{\text {traitées }}$ homme.mois. Ceci renforce l'argument de développer le compostage de proximité (au plus près de la production des déchets) et questionne sur la construction de grands sites.

Mots clés : Caractéristiques des déchets, échelle d'activité, productivité, compostage, Dschang

\section{Comparative study of the performance of Siteu and Ngui composting platforms in Dschang (Cameroon)}

\section{Ngahane Emilienne Laure, PhD}

Université de Dschang, Faculté d'Agronomie et des Sciences Agricoles, Filière des Métiers du Bois, de l'Eau et de l'Environnement

(UDs/FASA/FMBEE)

Sagne Moubé Joël, PhD

Université de Dschang, Faculté des Lettres et des Sciences Humaines,

Département de Géographie (UDs/FLSH/DG)

\section{Nana Paulin, PhD}

Université de Dschang, Faculté d'Agronomie et des Sciences Agricoles, Filière des Métiers du Bois, de l'Eau et de l'Environnement (UDs/FASA/FMBEE)

\section{Abstract}

Artisanal composting is the method of treating the waste's fermentable fraction in Dschang. Qualified as a high intensity workforce, it provides three 
direct jobs per ton of daily treated waste. Around 40 people work in the two nowadays operational platforms and treat an average of 3,000 ton of waste each year. To foresee their autonomy, the guarantee of the sustainability of activities and consolidation of these jobs, the teams were assessed using the workforce assessment method. It's a practical method for expressing the productivity of workers to express the cost of production and identify optimization levers. In this vein, the increase in the quantities of waste treated per agent in order to reduce the production cost and the improvement of the quality of the compost produced in order to facilitate its use have been identified as the internal levers. However, if it is readily accepted that the quality of compost is mainly conditioned by the nature of the initial waste; so is the quantity of compost obtained. In fact, the sorting stage as well as the screening stage, necessary to obtain good quality compost, also represent the most time-consuming stages and which objectively determine the worker's productivity. In Dschang, these two stages represent 84\% (66\% for sorting and $18 \%$ for screening). Ngui's team with a monthly productivity of 10.41 ton of waste treated/person performs $22 \%$ more than Siteu's team with a monthly productivity of 8.53 ton of waste treated/person. The major factors justifying these variations are the quality of incoming waste (at $46.2 \%$ ) and site development (at 34.3\%). Some negative feedback due to the presence of unwanted items were also recorded among users of the compost produced in Siteu. Therefore, due to the better quality of incoming waste and the concentration of activities on a limited space on the Ngui platform compared to that of Siteu, the consolidation of the jobs created and the sustainability of the composting activity would be ensured at Ngui. It was established that continuation of activities would require, among other things, the achievement of a minimum monthly productivity of 10 ton of waste treated/person. This strengthens the argument for developing local composting (as close as possible to waste production) and raises questions about the construction of large sites.

Keywords: Waste's characteristics, activity scale, productivity, artisanal composting, Dschang

\section{Introduction}

Le développement du compostage à Dschang ainsi que la création d'une entité opérationnelle qui porterait cette activité ont justifié l'impérativité de la connaissance précise du coût de production (constitué essentiellement du coût de main d'œuvre (Garnier et al., 2014)) et la nécessité de définir les actions stratégiques d'optimisation de l'ensemble des ressources disponibles afin d'assurer son autonomisation. En effet, l'autonomisation des structures partenaires et la valorisation de l'expérience acquise est l'un des trois sousobjectifs spécifiques du Programme Africompost (Guillaume et al., 2014) 
auquel sont inscrites les plateformes de compostage de Dschang. L'analyse coûts-bénéfices de la filière a révélé en 2019 que :

- Traiter une tonne de déchets par compostage à Dschang coûte en moyenne 12136 FCFA $(18,5 €)$ dont 8631 FCFA $(13,1 €)$ pour la production alors que les recettes de la vente du compost produit ne représentent que $4800 \mathrm{FCFA}(7,3 €)$. Ces coûts bien qu'inférieurs à ceux prospectés à Bouaké en présentent la même structure : $24,8 €$ pour le traitement d'une tonne de déchets dont 19,9 $€$ pour la production alors que la vente du compost obtenu ne rapporte que 12,2 $€$. (Ngahane et al., 2018).

- Les facteurs internes sur lesquels il faut s'appuyer pour diminuer ce coût sont l'augmentation de la productivité à 10 tonnes de déchets traités par agent et par mois au minimum et l'augmentation des tonnages traités à 5000 tonnes par an. Ceci correspond bien à ce qu'en 2014, Guillaume et al. avaient appelé l'accentuation de la responsabilisation des opérateurs locaux de compostage.

Les tableaux de bord techniques et financiers montrent que le site de Ngui est plus productif que celui de Siteu tant en quantité de déchets traités par agent qu'en qualité du compost produit et vendu. Les bilans main d'œuvre ont été réalisés afin de mesurer et d'analyser la productivité des équipes par étape et sous-étape et d'entrevoir objectivement les actions futures.

\section{Matériel et methodes}

\subsection{Présentation des plateformes de compostage}

Le compostage à Dschang se déroule au sein de deux plateformes dont les emplacements ont été stratégiquement pensés selon les possibilités techniques et les disponibilités foncières locales.

A Ngui, la plateforme d'une capacité de traitement de 2000 t/an s'étend sur $1848 \mathrm{~m}^{2}$ dont $820 \mathrm{~m}^{2}$ sous hangar. Elle absorbe les déchets frais directement pré-collectés dans les ménages de la zone et alentours afin d'assainir rapidement le quartier et limiter les quantités de déchets à transférer à la décharge finale. Aujourd'hui, le site fonctionne à sa capacité maximale et on y compte 29 agents composteurs et un chef de site. 


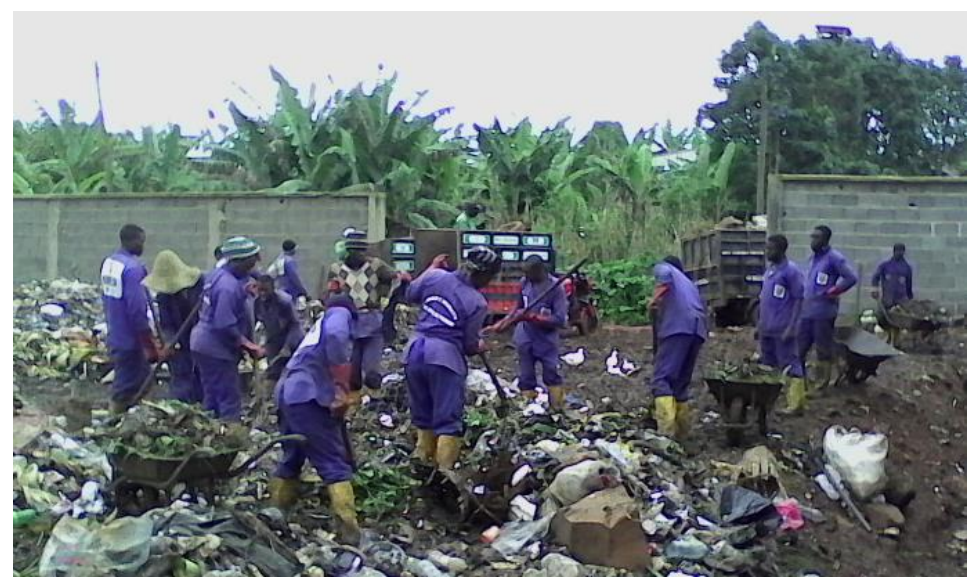

Figure 1. Tri grossier au sol sur la plateforme de Ngui (Source : Ngahane, 2020)

A Siteu, la plateforme d'une capacité de traitement de 9000 t/an s'étend sur environ $8000 \mathrm{~m}^{2}$ dont $2000 \mathrm{~m}^{2}$ sous hangar. Elle détourne une part des déchets collectés le long des grandes artères du centre-ville et au niveau des points de regroupement de la zone urbaine de Dschang. Il est à préciser qu'à Dschang, la production des déchets solides urbains est estimée à 25000 tonnes pour 2020. Aujourd'hui, l'équipe constituée de 19 agents composteurs et d'un chef de site traite environ 1500 tonnes de déchets par an.

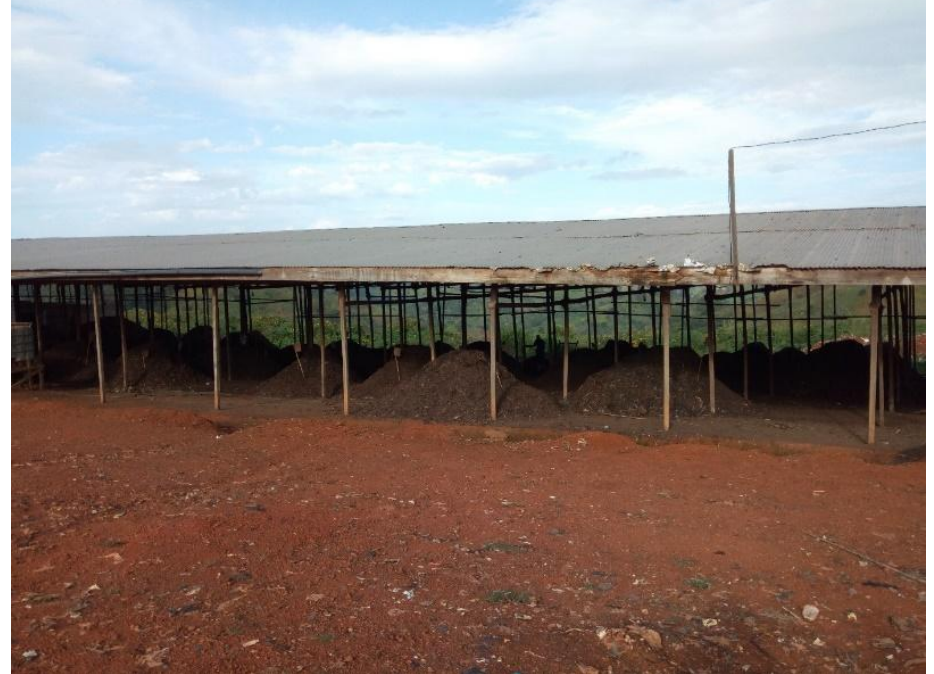

Figure 2. Vue d'ensemble de la plateforme de Siteu (Source : Garnier, 2018)

\subsection{Méthode de caractérisation des déchets entrants}

La caractérisation des déchets traités au sein des plateformes de compostage se fait tous les mois selon le protocole mis en place en 2012 par Gevalor dans le cadre du Programme Africompost. Ce protocole, en accord avec les normes AFNOR X30-408 Déchets ménagers et assimilés - 
Caractérisation d'un échantillon de déchets ménagers, a été validé par le Gold Standard pour le monitoring des émissions de carbone évitées et grâce auxquelles les plateformes bénéficient de la finance carbone depuis 2019. La méthode est directe et basée sur le tri manuel et la répartition des déchets en sept (7) catégories (Bois et sous-produits ; Papiers - cartons ; Nourriture, déchets de nourriture et autres putrescibles ; Textiles ; Déchets verts ; Inertes et Fines). La table de caractérisation comprend deux (2) coupures granulométriques permettant une catégorisation selon que le diamètre des déchets soit i) supérieur à $100 \mathrm{~mm}$, ii) entre 12 et $100 \mathrm{~mm}$ et iii) inférieur à 12 mm (fines). Les données obtenues permettent ensuite une classification grossière distinguant les compostables (Nourriture, déchets de nourriture et autres putrescibles ; Déchets verts ; 30\% des papiers-cartons ; 80\% des fines) des autres déchets. Aussi, l'aspect visuel et l'état général des déchets traités ont été considérés dans le sens où ils influencent la vitesse et la qualité du tri.

\subsection{Mesure des performances}

Les performances productives des équipes de Siteu et de Ngui à Dschang ont été mesurées en juillet 2018 et septembre 2020, respectivement.

Le bilan main d'œuvre se résume à la mesure de la productivité $(\mathrm{P})$ qui est définie comme le rapport entre une production et les ressources mises en œuvre pour l'obtenir. Dans le cas du compostage, la productivité représente le nombre d'agents et le temps qu'ils ont mis pour traiter des tonnes de déchets ou pour produire des tonnes de compost. Elle est exprimée soit en tonnes de

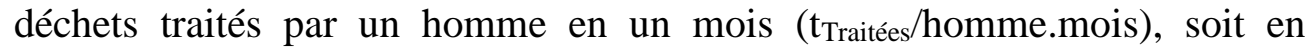
hommes par mois pour traiter une tonne de déchets (homme.mois/t $t_{\text {Traitée). Une }}$ valeur de productivité regroupe trois types de données : une quantité de temps (t), une quantité d'hommes (homme), une quantité d'activité (A).

Les bilans main d'œuvre à Dschang ont été réalisés en suivant le guide méthodologique élaboré par la plateforme Re-Sources et en l'adaptant aux spécificités locales. Les différentes étapes suivies sont :

- Le recensement des données initiales et les travaux préliminaires

Une fiche élaborée à cet effet a été remplie avant le début des investigations sur le terrain. Des informations préliminaires sur l'activité de compostage sur les sites y ont été renseignées, les données générales ont été fournies (rapport financier, tableau de bord technique, résultats du dernier bilan matière, résultats des caractérisations, etc.), le procédé de compostage a été schématisé pour en ressortir toutes les activités (étapes et sous-étapes). 


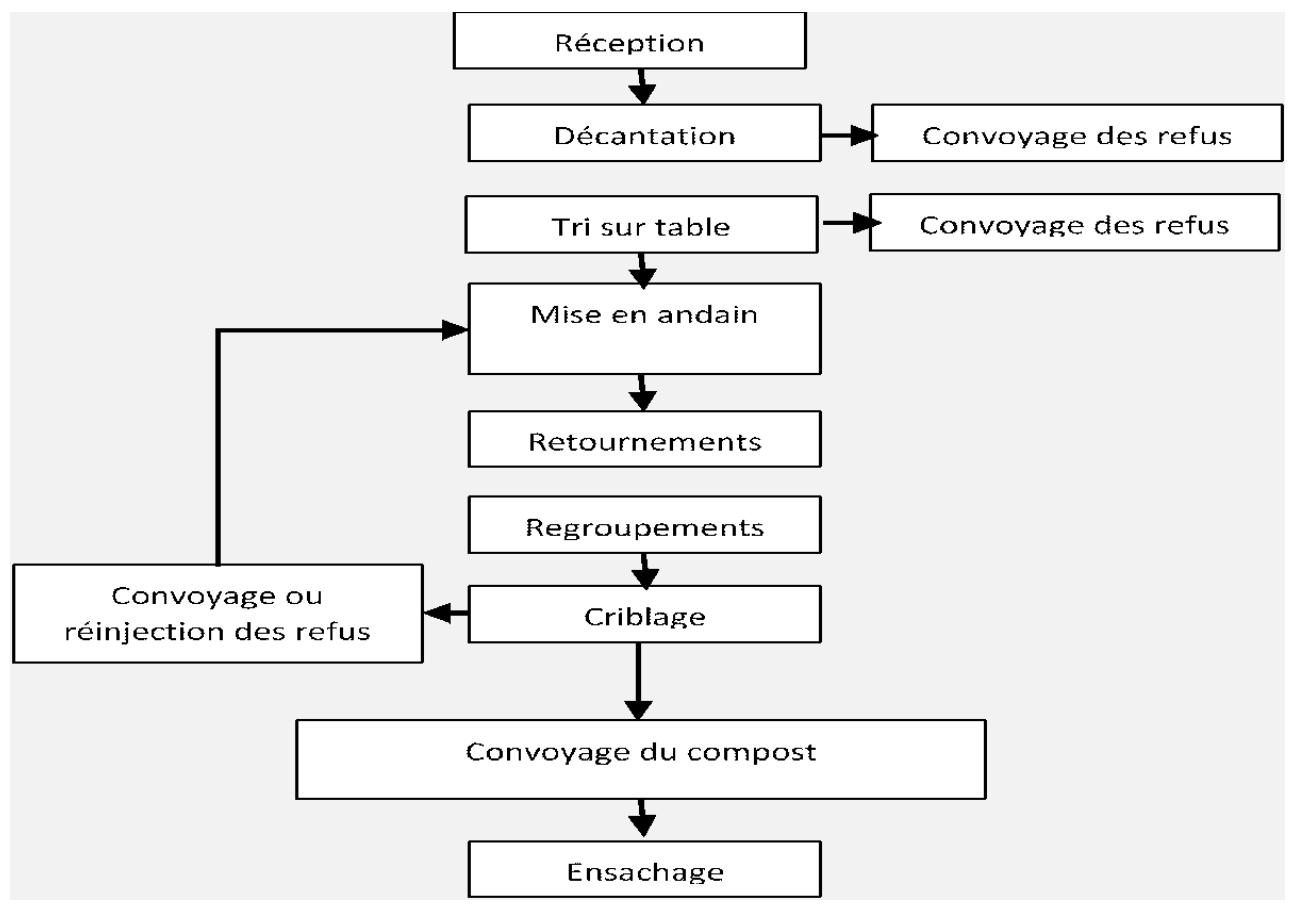

Figure 5. Représentation simplifiée du procédé de compostage à Dschang

Tableau 1. Etapes et sous-étapes du compostage à Dschang

\begin{tabular}{|c|c|}
\hline Etapes & Sous-étapes \\
\hline TRI & $\begin{array}{l}\text { Pré-tri ou Décantation } \\
\text { Tri sur table } \\
\text { Convoyage des refus } \\
\text { Convoyage de la FFOM (Fraction } \\
\text { Fermentescible des Ordures Ménagères) } \\
\text { et mise en andain }\end{array}$ \\
\hline RETOURNEMENTS & $\begin{array}{l}\text { Retournement } 1 \\
\text { Retournement } 2 \\
\text { Retournement } 3 \\
\text { Retournement } 4 \\
\text { Retournement } 5 \\
\text { Retournement } 6 \\
\end{array}$ \\
\hline REGROUPEMENTS & Regroupements \\
\hline CRIBLAGE & $\begin{array}{l}\text { Alimentation du plan incliné } \\
\text { Convoyage du compost } \\
\text { Convoyage des refus }\end{array}$ \\
\hline MISE EN SAC & $\begin{array}{l}\text { Remplissage des sacs } \\
\text { Pesée et emmagasinage des sacs } \\
\text { Couture des sacs }\end{array}$ \\
\hline ENTRETIEN & Entretien \\
\hline
\end{tabular}


- Les observations et mesures de terrain

Les journées d'observation ont été découpées en 3 parties égales de $2 \mathrm{~h} 15^{\prime}$ et la mobilisation des agents entre les différentes activités n'est représentative que d'une partie de la journée : 7h30-9h45, 9h45-12h00, 13h00$15 \mathrm{~h} 15$.

L'observation des activités s'est déroulée selon les calendriers des tableaux 2 et 3 .

Tableau 2. Calendrier des observations à Siteu

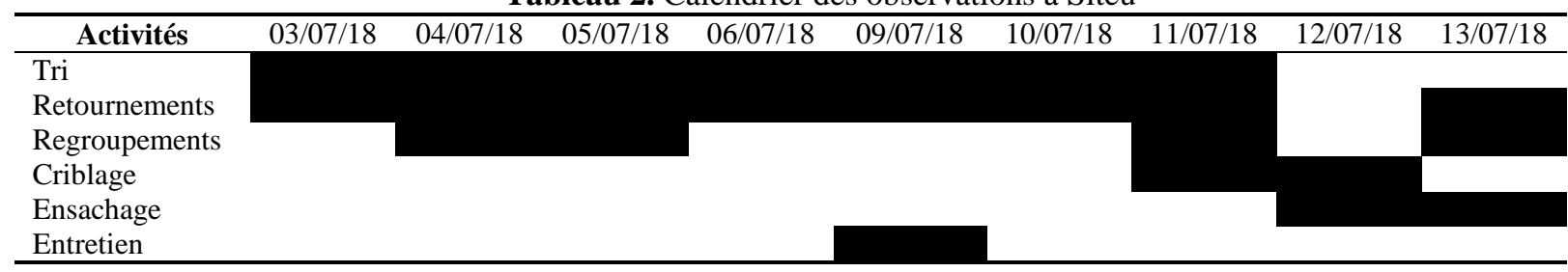

Tableau 3. Calendrier des observations à Ngui

\begin{tabular}{lllllll}
\hline \multicolumn{1}{c}{ Activités } & $21 / 09 / 20$ & $22 / 09 / 20$ & $23 / 09 / 20$ & $24 / 09 / 20$ & $25 / 09 / 20$ & $28 / 09 / 20$ \\
\hline Tri & & & & & & \\
Retournements & & & & & & \\
Regroupements & & & & & & \\
Criblage & & & & & & \\
Ensachage & & & & & & \\
Entretien & & & & & & \\
\hline
\end{tabular}

- La compilation des résultats

Un fichier Excel conçu à cet effet et comportant plusieurs onglets (présentation, données préalables, données du BMOE, résultats, lexique) a permis la compilation, le traitement automatique et la visualisation des résultats après le renseignement des différentes cellules par les données issues des observations et mesures de terrain.

\subsection{Méthode de comparaison des performances}

Pour chaque étape et sous-étape (activité, Ai), les performances (productivités, P) obtenues ont été comparées entre elles par la différence $\left(\Delta \mathrm{P}_{\mathrm{Ai}}\right)$ observée entre les deux sites (Ngui et Siteu).

$$
\Delta \mathbf{P}_{\text {Ai }}=\left|\mathbf{P}_{\text {Ai(Ngui) }}-\mathbf{P}_{\text {Ai(Siteu })}\right|
$$

Par la suite, la part explicative de chaque activité $\left(\dot{\mathrm{P}}_{\mathrm{Ai}}\right)$ sur la différence de productivité globale observée a été calculée en multipliant cette différence $\left(\Delta \mathrm{P}_{\mathrm{Ai}}\right)$ par la moyenne du temps de travail occupé $\left(\overline{\mathbf{x}} \mathrm{t}_{\mathrm{A}}\right)$ sur les deux sites par ladite activité.

$$
\begin{gathered}
\dot{\mathbf{P}}_{A i}=\Delta \mathbf{P}_{A i} * \overline{\mathbf{x}} \mathbf{t}_{\mathrm{Ai}} \\
\text { Où, } \overline{\mathbf{x}} \mathbf{t}_{\mathrm{Ai}}=1 / 2\left(\mathbf{t}_{\mathrm{Ai}(\mathrm{Ngui})}+\mathbf{t}_{\mathrm{Ai}(\mathrm{Siteu})}\right)
\end{gathered}
$$


Une analyse comparative plus détaillée des sous-étapes a permis de les classer en trois (3) groupes $\left(G_{1}, G_{2}\right.$ et $\left.G_{3}\right)$ en rapport avec leurs facteurs majeurs explicatifs des différences observées (caractéristiques des déchets entrants, aménagement du site et performances individuelles spécifiques). Enfin, la part explicative de chaque groupe $\left(\dot{\mathrm{P}}_{\mathrm{Gi}}\right)$ sur la différence globale observée a été calculée en sommant les parts explicatives des activités influencées par ce facteur.

$$
\dot{\mathbf{P}}_{\text {Gi }}=\sum \dot{\mathbf{P}}_{\text {AGi }}
$$

Tableau 4. Facteurs majeurs explicatifs des différences de performances observées

\begin{tabular}{lccc}
\hline \multicolumn{1}{c}{ SOUS-ETAPES } & $\begin{array}{c}\text { Groupe 1 : } \\
\text { Caractéristiques } \\
\text { de déchets }\end{array}$ & $\begin{array}{c}\text { Groupe 2 : } \\
\text { Aménagement } \\
\text { du site }\end{array}$ & $\begin{array}{c}\text { Groupe 3 : } \\
\text { Performances } \\
\text { individuelles } \\
\text { spécifiques }\end{array}$ \\
\hline I Pré-tri & $\mathrm{X}$ & $\mathrm{X}$ & \\
II Alimentation tri & $\mathrm{X}$ & $\mathrm{X}$ & \\
$\begin{array}{l}\text { III Tri sur table } \\
\text { IV Convoyage des refus } \\
\text { V Convoyage de la FFOM }\end{array}$ & $\mathrm{X}$ & $\mathrm{X}$ \\
\hline VI RETOURNEMENTS & $\mathrm{X}$ & $\mathrm{X}$ \\
\hline VII REGROUPEMENTS & $\mathrm{X}$ & $\mathrm{X}$ \\
\hline $\begin{array}{l}\text { VIII Convoyage du compost } \\
\text { IX Alimentation du plan incliné }\end{array}$ & $\mathrm{X}$ & $\mathrm{X}$ & \\
X Convoyage des refus & $\mathrm{X}$ & $\mathrm{X}$ & \\
\hline XI Remplissage des sacs & & $\mathrm{X}$ \\
XII Couture des sacs & $\mathrm{X}$ & $\mathrm{X}$ \\
XIII Pesée et emmagasinage sacs & $\mathrm{X}$ & \\
\hline XIV ENTRETIEN & $\mathrm{X}$ & $\mathrm{X}$ \\
\hline XV ENCADREMENT & &
\end{tabular}

Les travaux ont été bien préparés, leur réalisation s'est déroulée dans de bonnes conditions et les résultats obtenus ont été partagés, discutés, validés et aujourd'hui diffusés.

\section{Résultats et discussion}

\subsection{Caractéristiques générales des déchets traités}

Les caractéristiques des déchets traités dans les sites présentent des spécificités, lesquelles auraient une influence notable sur la qualité du compost et la productivité des agents. Ceci interpelle sur la définition d'objectifs communs de traitement par agent sur les deux plateformes. En effet, les déchets traités à Ngui proviennent de la pré-collecte domiciliaire tandis que ceux traités à Siteu sont issus de la collecte urbaine. 

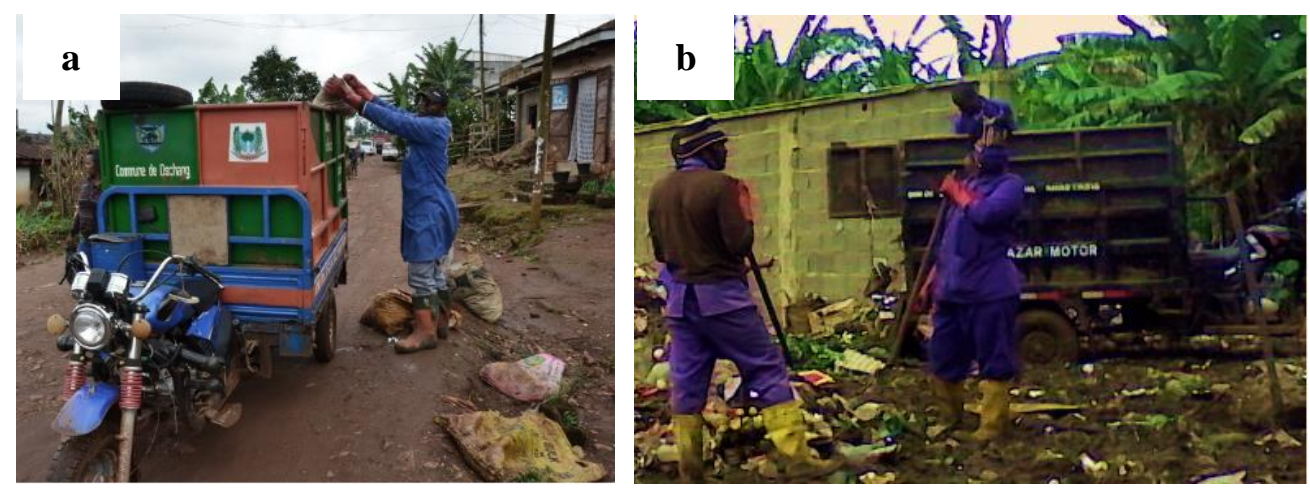

Figure 3a et 3b. Pré-collecte des déchets et approvisionnement de la plateforme de Ngui

(Source : Garnier, 2015 et Ngahane, 2020)

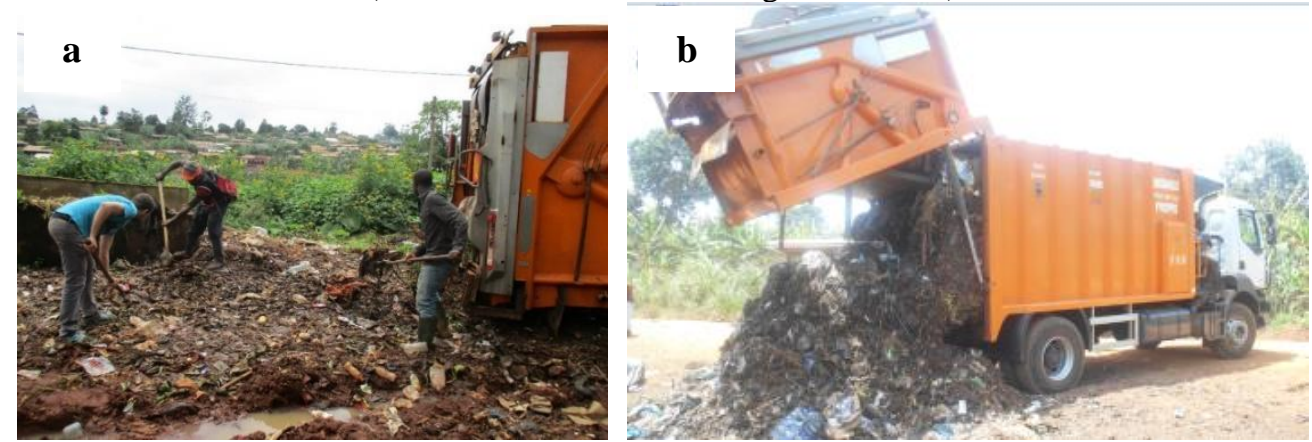

Figure 4a et 4b. Collecte des déchets et approvisionnement de la plateforme de Siteu (Source : Garnier, 2015)

\subsubsection{Composition des déchets traités}

Les déchets traités sur la plateforme de compostage de Ngui exclusifs aux activités ménagères contiennent un peu plus de matière organique et moins de textiles, papiers/cartons que ceux traités à Siteu où l'apport d'activités administratives, artisanales et commerciales est visible (Figure 6).

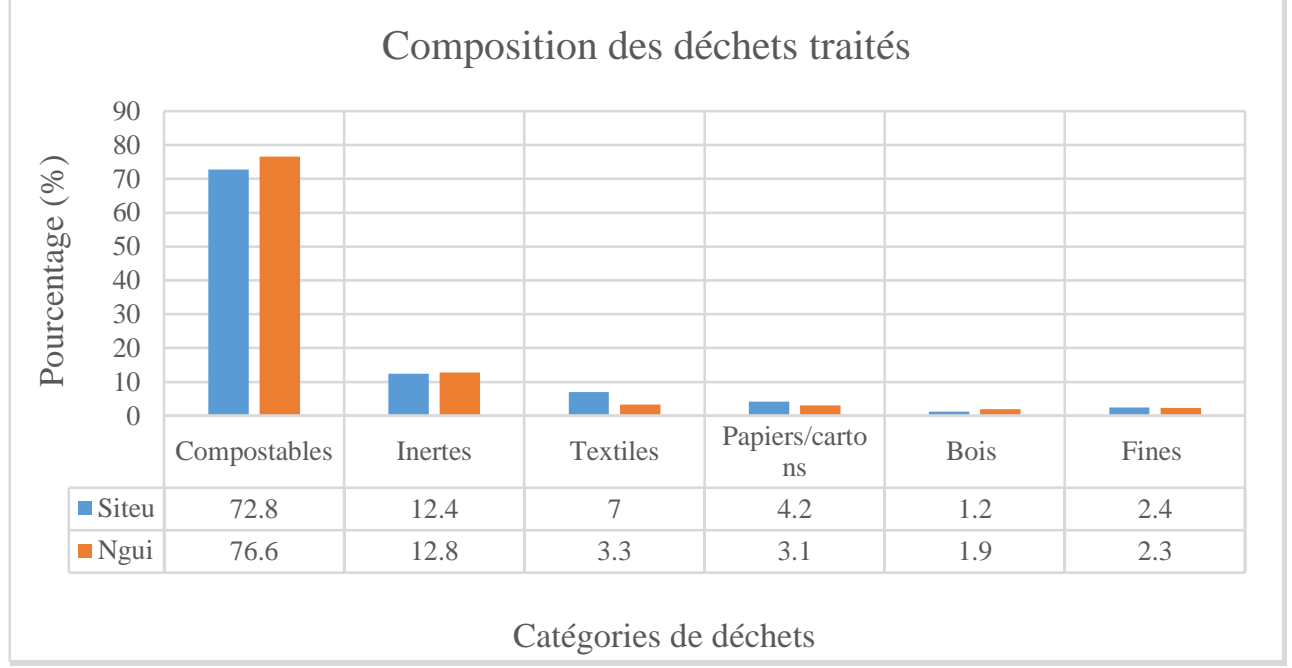

Figure 6. Composition des déchets traités sur les plateformes 


\subsubsection{Etat général des déchets entrants}

A Ngui, les ordures ménagères sont acheminées régulièrement à l'aide des tricycles affectés à la pré-collecte. Elles sont fraiches car produites au rythme des activités ménagères, conservées dans des contenants au sein des concessions et souvent à l'abri des intempéries et sur de courtes durées ( 2 jours en moyenne). Leur tassement est assez léger durant le parcours et leurs fines sont essentiellement organiques (restes alimentaires et terre végétale).

A Siteu, les déchets solides arrivent dans deux camions à compaction et deux camions bennes affectés à la collecte. Ceux-ci sont de fait compactés, de dégradation entamée et parfois très avancée, souvent souillés dans les dépotoirs ; leurs fines sont légèrement minérales (sables, poussières et boues).

Ceci pour dire que les déchets entrants à Ngui via la pré-collecte domiciliaire sont facilement manipulables et reconnaissables à l'œil, donc plus facilement traitables que ceux entrant à Siteu via la collecte urbaine.

\subsection{Performances des équipes de production}

\subsubsection{Récapitulatif des observations}

Les tableaux 5 et 6 donnent le récapitulatif des observations en équivalence :

Tableau 5. Tableau des observations en équivalence à Siteu

\begin{tabular}{lccc}
\hline \multicolumn{1}{c}{ Activités } & $\begin{array}{c}\text { Temps } \\
\text { d'observation } \\
\text { (éq Jours) }\end{array}$ & Production & $\begin{array}{c}\text { Agents mobilisés } \\
\text { (éq personnes) }\end{array}$ \\
\hline Tri & 7 & 59 tonnes de déchets triées & 94 \\
Retournements & $4 / 3$ & 20 retournements effectués & 7 \\
Regroupements & 1 & 10 regroupements effectués & 4 \\
Criblage & $4 / 3$ & 10 t criblées & 5 \\
Ensachage & $2 / 3$ & $(5 \mathrm{t}$ de compost obtenu) & 6 \\
Entretien & 1 & 5 te compost ensachées & 1 \\
\hline \multicolumn{1}{c}{ Activités } & Temps d'observation & Tout le site nettoyé & Production \\
\hline Tri & (éq Jours) & Agents mobilisés \\
Retournements & 4,3 & 30 retournements effectués & 10 \\
Regroupements & 2,72 & 10 regroupements effectués & 4 \\
Criblage & 1 & 10 t criblées & 4 \\
Ensachage & $4 / 3$ & $(4,5$ t de compost obtenu) & 6 \\
Entretien & $1 / 3$ & 4,5 te compost ensachées & 1 \\
\hline
\end{tabular}

\subsubsection{Productivité globale des agents}

A Siteu, la productivité totale observée durant la période est de $388 \mathrm{~kg}$ de déchets traités par homme et par jour, soit, $8,53 \mathrm{t}_{\text {traitées }}$ /homme.mois pour juillet 2018 avec 22 jours ouvrés. Le tri, le criblage et la mise en sac sont les 
étapes les plus chronophages avec $62 \%, 19 \%$ et $10 \%$ comme proportions respectives.

A Ngui, la productivité totale observée durant la période est de $473 \mathrm{~kg}$ de déchets traités par homme et par jour, soit, 10,41 $\mathrm{t}_{\text {traitées }} /$ homme.mois pour septembre 2020 avec 22 jours ouvrés. Le tri, le criblage et la mise en sac sont aussi les étapes les plus chronophages avec 69\%, 16\% et 6\% comme proportions respectives.

La productivité des agents à Ngui est supérieure de $22 \%$ à celle des agents à Siteu; la mise en sac, le criblage et les retournements expliquent grandement (à 61,5\%) cette différence avec 28\%, 17,5\% et 16\% comme proportions explicatives respectives.

Les performances observées par étape et la proportion qu'occupe chacune des grandes étapes dans cette productivité sont présentées dans les tableaux 7,8 et figure 7 .

Tableau 7. Productivité des agents composteurs par grande étape

\begin{tabular}{lcccc}
\hline & \multicolumn{2}{c}{$\begin{array}{c}\text { Productivité }(\mathrm{P}) \text { en } \\
\text { tonnes traitées/homme.j }\end{array}$} & \multicolumn{2}{c}{$\begin{array}{c}\text { Productivité }(\mathrm{P}) \text { en } \\
\text { homme.j/tonnes traitées }\end{array}$} \\
\cline { 2 - 5 } & Siteu & Ngui & Siteu & Ngui \\
\hline PRODUCTIVITE TOTALE & $\mathbf{0 , 3 8 8}$ & $\mathbf{0 , 4 7 3}$ & $\mathbf{2 , 5 8}$ & $\mathbf{2 , 1 1}$ \\
\hline Tri & 0,6 & 0,7 & 1,59 & 1,46 \\
Retournements & 29,0 & 44,8 & 0,03 & 0,02 \\
Regroupements & 25,4 & 37,3 & 0,04 & 0,03 \\
Criblage & 2,1 & 3,1 & 0,48 & 0,33 \\
Mise en sac (ensachage) & 4,0 & 7,5 & 0,25 & 0,13 \\
Entretien & 31,8 & 40,4 & 0,03 & 0,02 \\
Encadrement & 6,6 & 8,4 & 0,15 & 0,12 \\
\hline
\end{tabular}

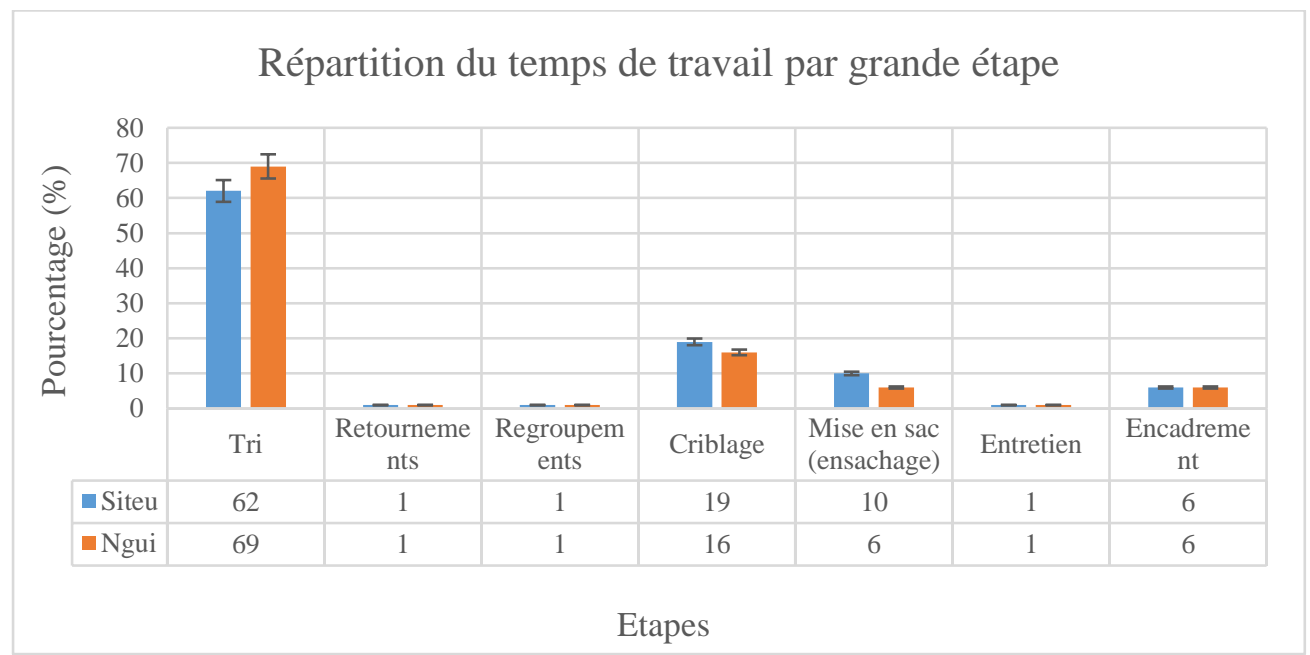

Figure 7. Répartition du temps passé par grande étape 
Tableau 8. Part explicative des grandes étapes sur la différence de productivité observée

\begin{tabular}{lccccc}
\hline & \multicolumn{3}{c}{ Productivité (P) en tonnes } & \multicolumn{2}{c}{ Proportion $(\%)$} \\
\cline { 2 - 6 } & traitées/h.j & Ngui & $\Delta \mathrm{P}$ & $\overline{\mathbf{x}} \mathrm{c}$ & $\dot{\mathbf{P}}$ \\
\hline PRODUCTIVITE & $\mathbf{0 , 3 8 8}$ & $\mathbf{0 , 4 7 3}$ & $\mathbf{0 , 0 8 5}$ & $\mathbf{1 0 0}$ & $\mathbf{1 0 0}$ \\
TOTALE & 0,6 & 0,7 & 0,1 & 66 & $\mathbf{6 , 5}$ \\
\hline Tri & 29 & 44,8 & 15,8 & 1 & $\mathbf{1 5 , 8}$ \\
Retournements & 25,4 & 37,3 & 11,9 & 1 & $\mathbf{1 1 , 9}$ \\
Regroupements & 2,1 & 3,1 & 1 & 18 & $\mathbf{1 7 , 5}$ \\
Criblage & 4 & 7,5 & 3,5 & 8 & $\mathbf{2 8}$ \\
Mise en sac & 31,8 & 40,4 & 8,6 & 1 & $\mathbf{9}$ \\
Entretien & 6,6 & 8,4 & 1,8 & 6 & $\mathbf{1 1}$ \\
Encadrement & & & & & \\
\hline
\end{tabular}

A Dschang, le compostage est pourvoyeur d'emplois ; à Ngui $(2,11)$ comme à Siteu (2,58), il est bel et bien dans le sillage des 3 emplois directs créés par tonne de déchets traités par jour comme évoqué par le CEFREPADE en 2012. Les étapes de tri et de criblage occupent majoritairement les équipes (84\% du temps ici) avec $66 \%$ du temps en moyenne pour la seule étape de tri ; c'est la même tendance sur les autres plateformes de compostage du programme Africompost (Lomé, Mahajanga et Bouaké).

Toutefois, les performances globales sont meilleures pour l'équipe de Ngui comparativement à celle de Siteu. La quantité de compost obtenu est liée à la productivité des agents et tout comme sa qualité, est surtout conditionnée par la nature des produits initiaux donc, par les caractéristiques des déchets entrants. En effet, les critiques sur la qualité médiocre du compost avaient déjà failli condamner le tri-compostage en liant cette mauvaise performance à la nature des déchets ménagers entrants (Aboulam, 2005).

D'autres facteurs entrent en jeu dans la différence des performances ; il s'agit de la taille du site (éloignement des ateliers de travail les uns des autres lié au niveau d'exploitation) et des performances individuelles spécifiques.

\subsubsection{Productivité des agents par sous-étape}

Un zoom sur les performances observées dans les sous-étapes révèle que :

- A Siteu comme à Ngui, le tri sur tables, le pré-tri (tri grossier au sol), l'alimentation du plan incliné lors du criblage et l'alimentation des tables de tri sont les plus chronophages (63\%) avec 30\%, 14\%, $10 \%$ et 9\% comme proportions moyennes respectives.

- Les facteurs relatifs aux caractéristiques des déchets expliquent à 46,2\% la différence de performance observée entre les deux plateformes en termes de productivité totale des agents alors que ceux relatifs au niveau d'exploitation des sites l'expliquent à $34,3 \%$. 
Les performances observées par sous-étape et la proportion qu'occupe chacune des sous-étapes dans cette productivité sont présentées dans les tableaux 9,10 et figure 8 .

Tableau 9. Productivité des agents composteurs par sous-étape

\begin{tabular}{|c|c|c|c|c|c|}
\hline & & \multicolumn{2}{|c|}{$\begin{array}{l}\text { Productivité }(\mathrm{P}) \text { en } \\
\text { tonnes } \\
\text { traitées/homme.j }\end{array}$} & \multicolumn{2}{|c|}{$\begin{array}{c}\text { Productivité }(\mathrm{P}) \text { en } \\
\text { homme.j / tonnes traitées }\end{array}$} \\
\hline & & Siteu & Ngui & Siteu & Ngui \\
\hline \multicolumn{2}{|c|}{ PRODUCTIVITE TOTALE } & $\mathbf{0 , 3 8 8}$ & 0,473 & 2,58 & 2,11 \\
\hline \multirow{5}{*}{ TRI } & I Pré-tri & 3,6 & 2,9 & 0,28 & 0,34 \\
\hline & II Alimentation tri & 4,5 & 4,2 & 0,22 & 0,24 \\
\hline & III Tri & 1,3 & 1,7 & 0,80 & 0,59 \\
\hline & IV Convoyage des refus de tri & 5,5 & 6,2 & 0,18 & 0,16 \\
\hline & $\begin{array}{l}\mathrm{V} \text { Acheminement FFOM et } \\
\text { formation des andains }\end{array}$ & 8,4 & 8,3 & 0,12 & 0,12 \\
\hline \multicolumn{2}{|c|}{ VI RETOURNEMENTS } & 29,0 & 44,8 & 0,03 & 0,02 \\
\hline \multicolumn{2}{|c|}{ VII REGROUPEMENTS } & 25,4 & 37,3 & 0,04 & 0,03 \\
\hline \multirow{3}{*}{ CRIBLAGE } & $\begin{array}{l}\text { VIII Acheminement du } \\
\text { compost }\end{array}$ & 8,3 & 12,2 & 0,12 & 0,08 \\
\hline & $\begin{array}{l}\text { IX Alimentation du plan } \\
\text { incliné }\end{array}$ & 4,1 & 6,1 & 0,24 & 0,16 \\
\hline & X Acheminement des refus & 8,3 & 12,2 & 0,12 & 0,08 \\
\hline \multirow{3}{*}{$\begin{array}{l}\text { MISE } \\
\text { SAC }\end{array}$} & XI Mise en sac du compost & 10,1 & 17,2 & 0,10 & 0,06 \\
\hline & XII Couture des sacs & 50,7 & 86,2 & 0,02 & 0,01 \\
\hline & $\begin{array}{l}\text { XIII Pesée-Emmagasinage de } \\
\text { sacs }\end{array}$ & 7,6 & 15,6 & 0,13 & 0,06 \\
\hline \multicolumn{2}{|c|}{ XIV ENTRETIEN } & 31,8 & 40,4 & 0,03 & 0,02 \\
\hline \multicolumn{2}{|c|}{ XV ENCADREMENT } & 6,6 & 8,4 & 0,15 & 0,12 \\
\hline
\end{tabular}




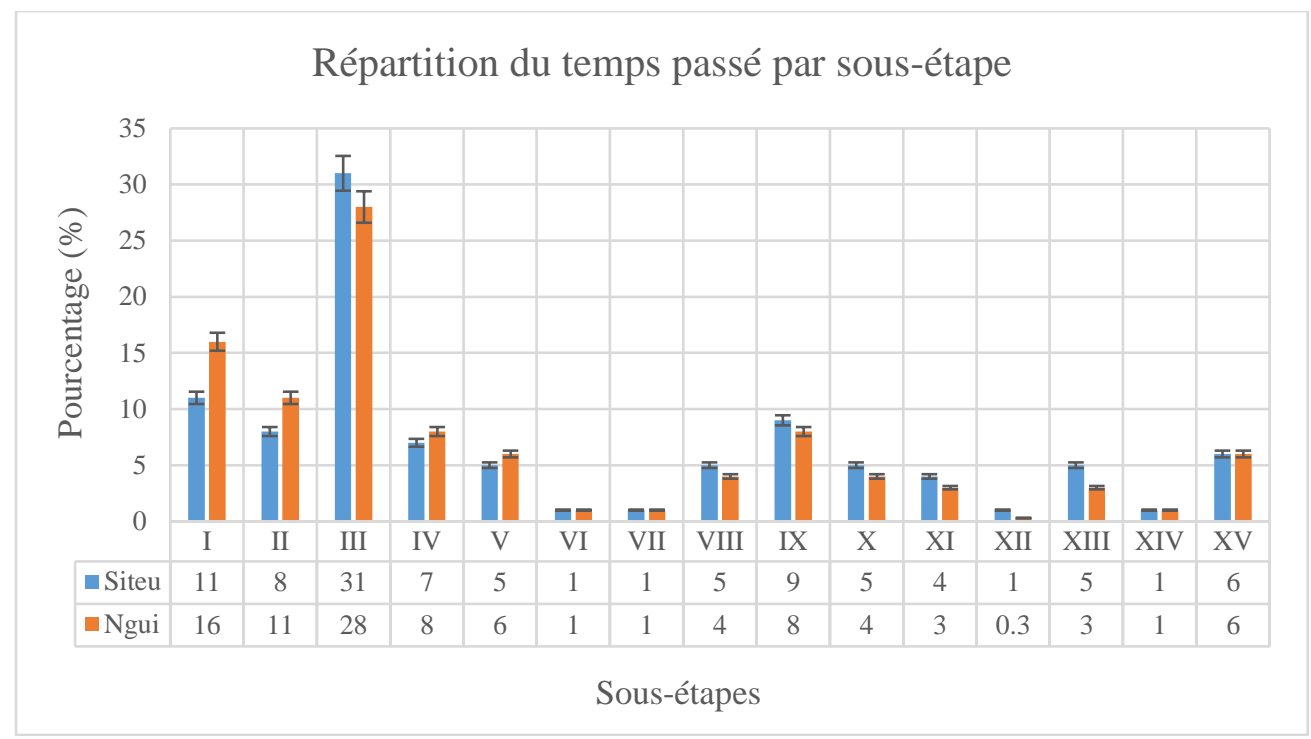

Figure 8. Répartition du temps passé par sous-étape

Tableau 10. Part explicative des sous- étapes sur la différence de productivité observée Productivité $(\mathrm{P})$ en tonnes traitées/homme.j

\begin{tabular}{lccccc}
\hline & Siteu & Ngui & $\Delta \mathrm{P}$ & $\overline{\mathbf{x}} \mathrm{c}$ & $\mathbf{\mathbf { P }}$ \\
\hline PRODUCTIVITE & $\mathbf{0 , 3 8 8}$ & $\mathbf{0 , 4 7 3}$ & $\mathbf{0 , 0 8 5}$ & $\mathbf{1 0 0}$ & $\mathbf{1 0 0}$ \\
TOTALE & 3,6 & 2,9 & 0,7 & 14 & $\mathbf{2 , 1}$ \\
\hline Pré-tri & 4,5 & 4,2 & 0,3 & 10 & $\mathbf{0 , 9}$ \\
Alimentation tri & 1,3 & 1,7 & 0,4 & 30 & $\mathbf{1 , 2}$ \\
Tri sur tables & 5,5 & 6,2 & 0,7 & 8 & $\mathbf{2 , 1}$ \\
Convoyage refus & 8,4 & 8,3 & 0,1 & 6 & $\mathbf{0 , 3}$ \\
Acheminement FFOM & 29 & 44,8 & 15,8 & 1 & $\mathbf{1 5 , 8}$ \\
RETOURNEMENTS & 25,4 & 37,3 & 11,9 & 1 & $\mathbf{1 1 , 9}$ \\
REGROUPEMENTS & 8,3 & 12,2 & 3,9 & 5 & $\mathbf{7}$ \\
Alimentation plan incliné & 4,1 & 6,1 & 2 & 9 & $\mathbf{3 , 6}$ \\
Convoyage compost & 8,3 & 12,2 & 3,9 & 5 & $\mathbf{7}$ \\
Convoyage refus & 10,1 & 17,2 & 7,1 & 4 & $\mathbf{3 , 9}$ \\
Remplissage des sacs & 50,7 & 86,2 & 35,5 & 1 & $\mathbf{1 9 , 6}$ \\
Couture des sacs & 7,6 & 15,6 & 8 & 4 & $\mathbf{4 , 4}$ \\
Pesée et emmagasinage & 31,8 & 40,4 & 8,6 & 1 & $\mathbf{9}$ \\
ENTRETIEN & 6,6 & 8,4 & 1,8 & 6 & $\mathbf{1 1}$ \\
ENCADREMENT & & & & & \\
\hline
\end{tabular}

A Siteu, l'état plus compacté des déchets entrants a influencé négativement les performances des agents dans les sous-étapes que sont le prétri, les retournements et regroupements, l'alimentation du plan incliné lors du criblage et le remplissage des sacs qui nécessitent dès lors plus d'efforts et de temps pour la décompaction, l'aération des andains, l'émottage de la matière organique durant le compostage et le criblage. L'état de dégradation avancée 
des déchets arrivant à Siteu a quant à lui influencé négativement les performances dans les sous-étapes de pré-tri et de tri sur tables par la difficulté qu'ont les agents à reconnaitre et à séparer la fraction fermentescible des autres fractions (indésirables). La grande emprise foncière du site alors dimensionné pour traiter 9000 tonnes de déchets par an mais ne fonctionnant qu'à $20 \%$ a été défavorable du fait de l'éloignement des ateliers de travail les uns des autres augmentant ainsi les distances à parcourir.

A Ngui, le fonctionnement du site à sa capacité maximale (concentration des activités sur un espace restreint et proximité des ateliers les uns des autres) a influencé positivement les performances des agents par le raccourcissement des distances à parcourir dans les sous-étapes qui impliquent le transport et le déplacement de la matière que sont l'alimentation des tables de tri, les regroupements avec déplacement du tas, le convoyage des refus (de tri et de criblage) et du compost, l'emmagasinage des sacs de compost. Aussi, l'alignement des ateliers suivant la configuration du site (dans le sens la pente) facilite les déplacements car il est plus aisé de descendre avec une brouette chargée et de monter avec une brouette vide que l'inverse. Ceci vient soutenir l'option de créer, à la place d'une seule unité centralisée, un certain nombre de petites unités dans différents points de la zone de production des déchets (CEFREPADE, 2012).

Tableau 11. Part des facteurs explicatifs de la différence de performances observée

\begin{tabular}{|c|c|c|c|}
\hline SOUS-ETAPES & $\begin{array}{c}\dot{\mathbf{P}}_{\mathrm{G1}}: \\
\text { Caractéristiques } \\
\text { de déchets }\end{array}$ & $\begin{array}{c}\dot{\mathbf{P}}_{\mathrm{G} 2}: \\
\begin{array}{l}\text { Aménagement } \\
\text { du site }\end{array}\end{array}$ & $\begin{array}{c}\dot{\mathbf{P}}_{\mathrm{G} 3}: \\
\text { Performances } \\
\text { individuelles } \\
\text { spécifiques }\end{array}$ \\
\hline Pré-tri & 2,1 & & \\
\hline Alimentation table de tri & & 0,9 & \\
\hline Tri sur table & 1,2 & & \\
\hline Convoyage des refus & & 2,1 & \\
\hline Convoyage de la FFOM & & 0,3 & \\
\hline RETOURNEMENTS & 16 & & \\
\hline REGROUPEMENTS & 6 & 6 & \\
\hline Alimentation du plan incliné & 7 & & \\
\hline Convoyage du compost & & 3,6 & \\
\hline Convoyage des refus & & 7 & \\
\hline Remplissage des sacs & 3,9 & & \\
\hline Couture des sacs & & & 19,6 \\
\hline Pesée et emmagasinage sacs & & 4,4 & \\
\hline ENTRETIEN & 4,5 & 4,5 & \\
\hline ENCADREMENT & 5,5 & 5,5 & \\
\hline TOTAL & 46,2 & $\mathbf{3 4 , 3}$ & 19,6 \\
\hline
\end{tabular}

Par ailleurs, les performances individuelles spécifiques à la sous-étape de «couture des sacs », expliquant à 19,6\% la différence globale observée, 
serait dû au fait qu'à Siteu, l'utilisation de la surfileuse était toute récente (2018) tandis qu'à Ngui, elle est maîtrisée par un agent qui l'utilise depuis 2016. Notons aussi que cette sous-étape a été mesurée une seule fois sur chaque site, il n'était donc pas possible de calculer une moyenne par site pour une comparaison objective, toutes choses qui remettent en cause sa pertinence dans cet exercice et en constituent une limite.

Il est à signaler qu'à Siteu, les performances enregistrées durant les travaux étaient meilleures que celles habituellement observées du fait de la meilleure organisation et de l'assiduité qu'imposaient la nature et l'ampleur de tels travaux. Les performances enregistrées à Ngui durant les travaux quant à elles étaient moindres du fait de la moins bonne qualité des déchets traités qu'habituellement $(61 \%$ de FFOM réellement compostés alors que la caractérisation annonçait 76\% de FFOM) et de l'embourbement du site suite aux aménagements nouvellement effectués. Ceci vient conforter l'idée locale selon laquelle la viabilité du compostage à Ngui serait assurée si la pré-collecte suivait.

\section{Conclusion}

A Dschang, bien que l'activité de compostage soit encore rythmée par celle de l'approvisionnement des sites en déchets, la plateforme de Ngui est plus performante que celle de Siteu. Les résultats obtenus au terme de cette analyse comparative des performances mesurées au sein des deux plateformes en donnent les facteurs explicatifs majeurs. La productivité sur la plateforme de compostage de Siteu est en deçà de la productivité attendue (10

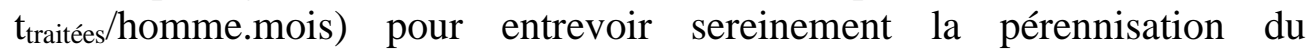
compostage sur ce site et la consolidation des emplois créés. Ceci renforce l'argumentaire du compostage de proximité (au plus près de la production des déchets) et questionne sur la construction de grands sites (changement d'échelle et performance en fonction de la taille des sites). Dans l'immédiat, cela interpelle sur la définition des objectifs communs de traitement aux deux sites et de qualité de compost produit. Etant donné que la pré-collecte ne saurait à l'heure actuelle alimenter la plateforme de Siteu en déchets du fait de la légèreté des tricycles, du fort relief et de l'éloignement de Siteu, il serait judicieux de penser et d'analyser dès à présent des scénarii pour la viabilité de l'activité à Siteu: collecte sélective des déchets propices au compostage (marchés, hôtels, restaurants et ménages effectuant le tri à la source) ; production du criblé de décharge directement sur la décharge contigüe à la plateforme exclusivement ou en plus du compostage ; etc.

\section{Remerciements}

Cet article est lié au projet Africompost-Dschang dont nous remercions toute personne physique ou morale ayant accompagné de près ou de loin, 
directement ou indirectement la mise en œuvre. Spécifiquement, nous remercions l'opérateur local de compostage à Dschang (ERA-CAMEROUN), ses partenaires techniques (Gevalor, Gret, l'Agence municipale de gestion des déchets de Dschang, l'Université de Dschang, l'Etablissement Providence), ses partenaires opérationnels (associations de pré-collecte Tockem, Cepdel et Adecotec) et ses partenaires financiers (l'Union Européenne, l'Agence Française de Développement, le Fonds Français pour l'Environnement Mondial, le SYCTOM de Paris). Nous n'oublions pas de saluer les apports des membres de la Faculté d'Agronomie et des Sciences Agricoles de l'université de Dschang.

\section{References:}

1. Aboulam S. (2005). Recherche d'une méthode d'analyse du fonctionnement des usines de tri-compostage des déchets ménagers. Fiabilité des bilans matière. Thèse de doctorat. INP-ENSAT. 123 p.

2. Biaou C. I., Hedible S. C., Landeou R. C. \& Boko M. (2019). Impact des Décharges Sauvages des Déchets Solides sur les Sols à Cotonou. European Scientific Journal, ESJ, 15(30), pp 94-104. Retrieved from https://eujournal.org/index.php/esj/article/view/12526, 23/04/2021

3. CEFREPADE (2012). Compostage des déchets ménagers dans les pays en développement - Modalités de mise en place et de suivi d'installations décentralisées pérennes. $57 \mathrm{p}$.

4. Etsè A., Sanonka T., Kokou S., Magnoudéwa B. B., Kokou D., Koffi K. A., Gado T. \& Gnon, B. (2014). Etude de la disponibilité du phosphore assimilable des composts des déchets urbains dans deux sols différents. European Scientific Journal, ESJ, 10(6), pp 156-167. Retrieved from https://doi.org/10.19044/esj.2014.v10n6p\%p, 23/04/2021

5. Garnier J. \& Njinoh B. (2015). Diagnostic de la filière DSM de la commune de Dschang. Rapport, $65 \mathrm{p}$.

6. Garnier J., Marciniak R. \& Delarue J. (2014). Analyse coûts-bénéfices de l'intégration du compostage dans la filière de gestion des déchets de Lomé. Rapport de synthèse, $15 \mathrm{p}$.

7. Guillaume M., Mercier J-R., Njietcheu M., Rakotonirina A. \& Tsitsikalis A. (2014). Evaluation à mi-parcours du Programme Africompost. Rapport de synthèse, Gevalor, ETC Terra et Fondation GoodPlanet, $17 \mathrm{p}$.

8. Ngahane E. L., Garnier J., Bromblet H. \& Vanié C. (2018). Axes stratégiques pour la pérennisation d'unités de compostage en Afrique: Cas de Bouaké en Côte d'Ivoire. Déchets Sciences et Techniques, $\mathrm{n}^{\circ} 76$, pp 43-51. 
9. Ngahane E. L. \& Garnier J. (2018). Synthèse de l'analyse coûtsbénéfices du compostage à Dschang. Document Africompost, 8p.

10. Ngnikam E., Vermande P. \& Rousseaux P., (1993). Traitement des déchets urbains. Une unité de compostage des ordures ménagères dans un quartier à habitat spontané à Yaoundé - Cameroun. Cahiers Agriculture, $\mathrm{n}^{\circ}$, pp 264-269.

11. Tahraoui Douma (2013). Valorisation par compostage des résidus solides urbains de la commune de Chlef, Algérie. Thèse de doctorat. Université de Limoges, Ecole Doctorale Sciences et Techniques, Groupement de Recherche Eau Sol Environnement, 244p.

12. Tape A. S. B., Coulibaly A., Anoh P. K. \& Aloko J. N. (2019). Production des Déchets et Santé des Travailleurs: Cas de la Mine d'or de Tongon (Côte d'Ivoire). European Scientific Journal, ESJ, 15(32), pp 104-118. Retrieved from https://doi.org/10.19044/esj.2019.v15n32p104, 23/04/2021

13. Tauvel Maud. Le traitement bio-mécanique des déchets : Avantages, inconvénients, coûts et jeux d'acteurs. Série Synthèse $\mathrm{N}^{\circ} 06-\mathrm{S} 01$, ENGREF Centre de Montpellier, 31p.

14. Toundou O., Tozo K., Amouzouvi K. A. A., Kolani L., Tchangbedji G., Kili K. \& Gnon B. (2014). Effets de la biomasse et du compost de Cassia Occidentalis L. sur la croissance en hauteur, le rendement du maïs (Zea Mays L.) et la teneur en NPK d'un sol dégradé en station expérimentale. European Scientific Journal, ESJ, 10(3), pp 294-308. Retrieved from https://doi.org/10.19044/esj.2014.v10n3p\%p, 23/04/2021 